\title{
ABSTRACT PARABOLIC SYSTEMS AND REGULARIZED SEMIGROUPS
}

\author{
QuAn Zheng And Yongsheng Li
}

The main purpose of this paper is to obtain the wellposedness of abstract parabolic systems in the sense of Petrovsij and Shilov under sharper conditions by using regularized semigroups. We also consider these systems with time-dependent coefficients, and give the applications to the corresponding parabolic systems on many function spaces.

\section{Introduction.}

Let $i A_{j}(1 \leq j \leq n)$ be commuting generators of bounded $C_{0^{-}}$-groups on a Banach space $X$, and write $A=\left(A_{1}, \ldots, A_{n}\right)$ and $A^{\mu}=A_{1}^{\mu_{1}} \cdots A_{n}^{\mu_{n}}$ for $\mu=\left(\mu_{1}, \ldots, \mu_{n}\right) \in \mathbf{N}_{0}^{n}$, where $\mathbf{N}_{0}=\mathbf{N} \cup\{0\}$. In this paper, we consider the system of differential equations

$$
\vec{u}^{\prime}(t)=\left(p_{j k}(A)\right) \vec{u}(t)(t>0), \quad \vec{u}(0)=\vec{u}_{0}
$$

on $X^{N}$, where $P(\xi) \equiv\left(p_{j k}(\xi)\right)$ is an $N \times N$ matrix of polynomials of $\xi \in \mathbf{R}^{n}$. We also can write $P(\xi)=\sum_{|\mu|<m} P_{\mu} \xi^{\mu}$, where $m \equiv \max \left\{\right.$ degree of $\left.p_{j k}\right\}$ is the degree of $P(\xi)$, and $P_{\mu} \in M_{N}(\mathbf{C})$ (the ring of $N \times N$ matrices over $\mathbf{C}$ ). Then $P(A) \equiv \sum_{|\mu| \leq m} P_{\mu} A^{\mu}$ with maximal domain is closable on $X^{N}$ (cf. $[4$, Theorem 14.1]).

By choosing $A=D \equiv-i\left(\frac{\partial}{\partial x_{1}}, \ldots, \frac{\partial}{\partial x_{n}}\right)$ one finds that (1.1), in fact, gives an abstract form of the system of differential equations

$$
\vec{u}^{\prime}(t)=\left(p_{j k}(D)\right) \vec{u}(t)(t>0), \quad \vec{u}(0)=\vec{u}_{0}
$$

on many function spaces. It is well known that a classical method to treat the wellposedness of (1.2) is to show $P(D)$ as the generator of a $C_{0}$-semigroup. However, many $P(D)$ do not generate a $C_{0}$-semigroup. An important result states that when the numerical range of $P(\xi)$ is contained in a left halfplane, $P(D)$ generates a $C_{0}$-semigroup on $\left(L^{p}\right)^{N}$ if and only if $p=2$. In particular, the Schrödinger operator $i \Delta$ on $L^{p}$ generates a $C_{0}$-semigroup if and only if $p=2[\mathbf{1 3}]$. Another simple example is that the wave equation on $L^{p}(p \neq 2$ and $n \neq 1)$, written as a system on $\left(L^{p}\right)^{n+1}$, cannot be treated by $C_{0}$-semigroups $[\mathbf{1 0}]$. 
Starting from this situation, people recently paid attention to approaching (1.1) and (1.2) by means of two important generalizations of $C_{0}$-semigroups, i.e., integrated and regularized semigroups $[2,3,4,10,11,12]$. More precisely, Hieber [10] first showed that some elliptic system (1.2) can be treated by integrated semigroups. The application of integrated semigroups to hyperbolic systems (1.2) appeared in [10, 11], while deLaubenfels [2] and Hieber et al [12] applied regularized semigroups to Petrovskij correct systems (1.1) and (1.2), respectively. Moreover, a Petrovskij correct system (1.2) with time-dependent coefficients was discussed by the simultaneous solution space (see [3]). However, based on the discussion in [24], it is known that the regularized semigroup is an appropriate tool for non-elliptic differential operators, while the effectiveness of the integrated semigroup is confined to the elliptic case. One of the reasons is that the resolvent set of the generator of an integrated semigroup must be nonempty (cf. [15]. It is known that this is often not the case for a matrix of differential operators, even for a differential operator.

It is well known that an important subclass of (1.1) is the so-called parabolic system in the sense of Shilov [9], while a special and degenerate case of that is the so-called parabolic and correct system in the sense of Petrovskij [9], respectively. Corresponding to the parabolic system (1.1) in the sense of Petrovskij we first show in $\S 2$ that $\overline{P(A)}$ generates an analytic semigroup. In $\S 3$ the parabolic system (1.1) in the sense of Shilov is dealt with using regularized semigroups. For Petrovskij correct systems we improve the corresponding results in [2]. The last section is concerned with time-dependent (1.1). The applications of our results to (1.2) and some concrete examples are given at the end of every section. Moreover, our results generalize the main results in [24] to the matrix case.

Throughout the paper, $B(X)$ will be the space of bounded linear operators on $X, \mathcal{S}$ (resp. $C_{c}^{\infty}$ ) the space of rapidly decreasing functions (resp. $C^{\infty}$ functions with compact support) on $\mathbf{R}^{n}$, and $H\left(\Delta_{\theta}, X\right)$ the set of analytic functions from $\Delta_{\theta}$ into $X$, where $\Delta_{\theta}=\{\lambda \in \mathbf{C} \backslash\{0\} ;|\arg \lambda|<\theta\}$ with $\theta \in(0, \pi / 2]$. By $D(B), R(B), \rho(B)$, and $R(\lambda, B)(\lambda \in \rho(B))$ we denote the domain, range, resolvent set, and resolvent of the operator $B$, respectively. We also denote by $B$ the operator $B I_{N}$ on $X^{N}$ with domain $(D(B))^{N}$, and $B\left(A^{\infty}\right)$ the Fréchet space $\left\{B: X \rightarrow D\left(A^{\infty}\right) \equiv \cap_{\mu \in \mathbf{N}_{0}^{n}} D\left(A^{\mu}\right) ; A^{\mu} B \in B(X)\right.$ for $\left.\mu \in \mathbf{N}_{0}^{n}\right\}$ with the family of seminorms $\|B\|_{\mu} \equiv\left\|A^{\mu} B\right\|$.

We now introduce a functional calculus for $i A_{j}(1 \leq j \leq n)$. Let $\mathcal{F}$ denote the Fourier transform. If $u \in \mathcal{F} L^{1}$, then there exists a unique $L^{1}$-function $\mathcal{F}^{-1} u$ (i.e., the inverse Fourier transform of $u$ in the distributional sense) 
such that $u=\mathcal{F}\left(\mathcal{F}^{-1} u\right)$. We define $u(A) \in B(X)$ by

$$
u(A) x=\int_{\mathbf{R}^{n}}\left(\mathcal{F}^{-1} u\right)(\xi) e^{-i(\xi, A)} x d \xi \quad \text { for } \quad x \in X .
$$

Define $M_{N}\left(\mathcal{F} L^{1}\right)=\left\{\left(u_{j k}\right) ; u_{j k} \in \mathcal{F} L^{1}\right\}$. Similarly, $M_{N}\left(L^{p}\right)$ and so on. If $u=\left(u_{j k}\right) \in M_{N}\left(\mathcal{F} L^{1}\right)$ then $u(A) \equiv\left(u_{j k}(A)\right) \in B\left(X^{N}\right)$. It is known that $M_{N}\left(\mathcal{F} L^{1}\right)$ is a (non-commutative) Banach algebra under matrix pointwise multiplication and addition with norm $\|u\|_{\mathcal{F} L^{1}} \equiv\left\|\mathcal{F}^{-1} u\right\|_{L^{1}}$, where $\mathcal{F}^{-1} u=\left(\mathcal{F}^{-1} u_{j k}\right)$. Furthermore, $u \mapsto u(A)$ is an algebra homomorphism from $M_{N}\left(\mathcal{F} L^{1}\right)$ into $B\left(X^{N}\right)$, and $\|u(A)\| \leq M\|u\|_{\mathcal{F} L^{1}}$ for all $u \in M_{N}\left(\mathcal{F} L^{1}\right)$ and some $M>0$.

The following lemma (cf. $[\mathbf{1 5}, \mathbf{1 9}, \mathbf{2 4}])$ will play an important role in our proofs.

\section{Lemma 1.1.}

(a) Let $E=\{\phi(A) x ; \phi \in \mathcal{S}$ and $x \in X\}$. Then $E \subset D\left(A^{\infty}\right), \bar{E}=X$, $\overline{\left.P(A)\right|_{E^{N}}}=\overline{P(A)}$, and $\phi(A) P(A) \subseteq P(A) \phi(A)=(P \phi)(A)$ for $\phi \in \mathcal{S}$.

(b) (Bernstein) If $n / 2<j \in \mathbf{N}$, then $H^{j}\left(\mathbf{R}^{n}\right) \subset \mathcal{F} L^{1}$ and there exists $M>0$ such that

$$
\|u\|_{\mathcal{F} L^{1}} \leq M\|u\|_{L^{2}}^{1-n / 2 j} \sum_{|\mu|=j}\left\|D^{\mu} u\right\|_{L^{2}}^{n / 2 j} \quad \text { for } u \in H^{j}\left(\mathbf{R}^{n}\right) .
$$

(c) Let $u_{t} \in C^{\infty}$ for $t \geq 0$. If there exist constants $M, L, a>0$ and $b \leq \frac{2 a}{n}-1$ such that $\left|D^{k} u_{t}(\xi)\right| \leq M\left(1+t^{|k|}\right)|\xi|^{b|k|-a}$ for $|\xi| \geq L, t \geq 0$, and $k \in \mathbf{N}_{0}^{n}\left(|k| \leq\left[\frac{n}{2}\right]+1\right)$, then there exist $M^{\prime}>0$ and $\psi \in C_{c}^{\infty}$ such that $u_{t}(1-\psi) \in \mathcal{F} L^{1}$ and $\left\|u_{t}(1-\psi)\right\|_{\mathcal{F} L^{1}} \leq M^{\prime}\left(1+t^{n / 2}\right)$ for $t \geq 0$.

Finally, let $|A|^{2}=\sum_{j=1}^{n} A_{j}^{2}$ and $C_{\alpha}=\left(1+|A|^{2}\right)^{-\alpha / 2}(\alpha \in \mathbf{R})$ as fractional powers. Then $C_{\alpha}=\left(1+|\cdot|^{2}\right)^{-\alpha / 2}(A) \in B(X)$ for $\alpha>0$ (see [5]), and $\left(R\left(C_{\alpha}\right)\right)^{N} \subseteq D(\overline{P(A)})$ for $\alpha>m$ (cf. [15, 24]). Relating to $\overline{P(A)}$ as the generator of a $C_{\alpha}$-regularized semigroup (see $\S 3$ below), we have the following result of wellposedness of (1.1) (cf. [4, 24]).

Lemma 1.2. Let $a \geq 0$, and $M_{\alpha}, \omega_{\alpha}$ be suitable constants depending on $\alpha$.

(a) If $\overline{P(A)}$ generates an (exponentially bounded) $C_{\alpha}$-regularized semigroup for every $\alpha>a$, then for every $\alpha>a$ and $\vec{u}_{0} \in\left(R\left(C_{\alpha+m}\right)\right)^{N}$, (1.1) has a unique solution $\vec{u}(t)$ (i.e., $\left.\vec{u} \in C\left([0, \infty), X^{N}\right) \cap C^{1}\left((0, \infty), X^{N}\right)\right)$ with $\|\vec{u}(t)\| \leq M_{\alpha} e^{\omega_{\alpha} t}\left\|C_{-\alpha} \vec{u}_{0}\right\|$ for $t \geq 0$.

(b) If $\overline{P(A)}$ generates an (exponentially bounded) differentiable $C_{\alpha}$-regularized semigroup for some $\alpha>a$, then for every $\vec{u}_{0} \in\left(R\left(C_{\alpha}\right)\right)^{N},(1.1)$ has a unique solution $\vec{u}(t)$ with $\|\vec{u}(t)\| \leq M_{\alpha} e^{\omega_{\alpha} t}\left\|C_{-\alpha} \vec{u}_{0}\right\|$ for $t \geq 0$. 


\section{Parabolic $P(\xi)$ in the sense of Petrovskij.}

In the sequel, we set $P_{m}(\xi)=\sum_{|\mu|=m} P_{\mu} \xi^{\mu}$ (the principal part of $P(\xi)$ ) and $\Lambda(P(\xi))=\sup _{1 \leq j \leq N} \operatorname{Re} \lambda_{j}(\xi)$ (the spectral bound of $P(\xi)$ ), where $\lambda_{j}(\xi)$ $(1 \leq j \leq N)$ are the eigenvalues of $P(\xi)$. From Friedman [8, p. 171], there exists a constant $M>0$ such that

$$
e^{t \Lambda(P(\xi))} \leq\left\|e^{t P(\xi)}\right\| \leq M\left(1+t+t|\xi|^{m}\right)^{N-1} e^{t \Lambda(P(\xi))} \quad \text { for } \xi \in \mathbf{R}^{n} \text { and } t \geq 0 .
$$

If $\Lambda\left(P_{m}(\xi)\right)<0$ for $\xi \in \mathbf{R}^{n} \backslash\{0\}$ then $P(\xi)$ is said to be parabolic in the sense of Petrovskij [9]. It is known that $m$ is always even for a parabolic system $P(\xi)$ in the sense of Petrovskij. In particular, the following characterizations hold.

Lemma 2.1. Let $\theta=\arctan \frac{\alpha}{\beta}$, where $\alpha=-\sup _{|\xi|=1} \Lambda\left(P_{m}(\xi)\right)$ and $\beta=$ $\sup _{|\xi|=1}\left|\Lambda\left(i P_{m}(\xi)\right)\right|$. Then the following statements are equivalent.

(a) $P(\xi)$ is parabolic in the sense of Petrovskij.

(b) There exist constants $\omega>0$ and $\omega^{\prime} \in \mathbf{R}$ such that $\Lambda(P(\xi)) \leq-\omega|\xi|^{m}+$ $\omega^{\prime}$ for $\xi \in \mathbf{R}^{n}$.

(c) There exist constants $\omega, M>0$ such that $\left\|e^{P(\xi)}\right\| \leq M \exp \left(-\omega|\xi|^{m}\right)$ for $\xi \in \mathbf{R}^{n}$.

(d) For every $\varepsilon \in(0, \theta)$, there exist constants $\omega_{\varepsilon}, M_{\varepsilon}, L_{\varepsilon}>0$ such that $\left\|e^{t P(\xi)}\right\| \leq M_{\varepsilon} \exp \left(-\omega_{\varepsilon}|\xi|^{m} \operatorname{Re} t\right)$ for $|\xi| \geq L_{\varepsilon}$ and $t \in \Delta_{\theta-\varepsilon}$.

Proof. The equivalence of (a) and (b) follows from [8, p. 191]. (d) $\Rightarrow$ (c) is obvious. (c) $\Rightarrow$ (b) follows easily from the first inequality in (2.1). It remains to show $(\mathrm{a}) \Rightarrow(\mathrm{d})$.

For any $\varepsilon \in(0, \theta)$, choose $\omega_{\varepsilon}=(\alpha-\beta \tan (\theta-\varepsilon)) / 3$. Then $\omega_{\varepsilon}>0$. Since the eigenvalues of $P_{m}(\xi)$ are all homogeneous, the parabolicity of $P(\xi)$ in the sense of Petrovskij implies that $\Lambda\left(P_{m}(\xi)\right) \leq-\alpha|\xi|^{m}$ and $\left|\Lambda\left(i P_{m}(\xi)\right)\right| \leq \beta|\xi|^{m}$ for $\xi \in \mathbf{R}^{n}$. It thus follows from the second inequality in (2.1) that

$$
\begin{aligned}
\left\|\exp \left(t P_{m}(\xi)\right)\right\| \leq & \left\|\exp \left(P_{m}(\xi) \operatorname{Re} t\right)\right\| \cdot\left\|\exp \left(i P_{m}(\xi) \operatorname{Im} t\right)\right\| \\
\leq & M\left(1+\operatorname{Re} t+\operatorname{Re} t|\xi|^{m}\right)^{N-1} \exp \left(\Lambda\left(P_{m}(\xi)\right) \operatorname{Re} t\right) \\
& \cdot\left(1+|\operatorname{Im} t|+|\operatorname{Im} t||\xi|^{m}\right)^{N-1} \exp \left(\left|\Lambda\left(i P_{m}(\xi)\right) \| \operatorname{Im} t\right|\right) \\
\leq & M_{\varepsilon}^{\prime}\left(1+\operatorname{Re} t+\operatorname{Re} t|\xi|^{m}\right)^{N-1} \exp \left(-3 \omega_{\varepsilon}|\xi|^{m} \operatorname{Re} t\right)
\end{aligned}
$$

for $\xi \in \mathbf{R}^{n}$ and $t \in \Delta_{\theta-\varepsilon}$. Also, there exists a constant $\omega>0$ such that

$$
\left\|t P(\xi)-t P_{m}(\xi)\right\| \leq \omega|t||\xi|^{m-1} \leq \omega_{\varepsilon}|\xi|^{m} \operatorname{Re} t \quad \text { for } \quad|\xi| \geq L_{\varepsilon} \text { and } t \in \Delta_{\theta-\varepsilon}
$$


where $L_{\varepsilon}=\omega \sec (\theta-\varepsilon) / \omega_{\varepsilon}$. Combining these two estimates yields

$$
\begin{aligned}
\left\|e^{t P(\xi)}\right\| & \leq\left\|\exp \left(t P_{m}(\xi)\right)\right\| \cdot\left\|\exp \left(t P(\xi)-t P_{m}(\xi)\right)\right\| \\
& \leq M_{\varepsilon}^{\prime}\left(1+\operatorname{Re} t+\operatorname{Re} t|\xi|^{m}\right)^{N-1} \exp \left(-2 \omega_{\varepsilon}|\xi|^{m} \operatorname{Re} t\right) \\
& \leq M_{\varepsilon} \exp \left(-\omega_{\varepsilon}|\xi|^{m} \operatorname{Re} t\right) \text { for }|\xi| \geq L_{\varepsilon} \text { and } t \in \Delta_{\theta-\varepsilon}
\end{aligned}
$$

Therefore the statement (d) holds.

We now can start with the main result of this section as follows.

Theorem 2.2. Let $P(\xi)$ be parabolic in the sense of Petrovskij and $\theta$ be defined in Lemma 2.1. Then $\overline{P(A)}$ generates an analytic semigroup $(T(t))_{t \in \Delta_{\theta}}$ on $X^{N}$, which satisfies the following statements.

(a) $\|T(t)\| \leq M\left(1+t^{N-1+n / 2}\right) e^{\omega t}$ for all $t \geq 0$ and some $M>0$, where $\omega=\sup _{\xi \in \mathbf{R}^{n}} \Lambda(P(\xi))$.

(b) $D(\overline{P(A)}) \subseteq\left(R\left(C_{\delta}\right)\right)^{N}$ for $\delta \in(0, m)$.

(c) $T(\cdot) \in H\left(\Delta_{\theta},\left(B\left(A^{\infty}\right)\right)^{N}\right)$.

Proof. By induction with respect to $|k|\left(k \in \mathbf{N}_{0}^{n}\right)$, we have that

$$
D^{k}\left(P(\xi)^{l} e^{t P(\xi)}\right)=\sum_{j=j_{0}}^{|k|} t^{j} Q_{j}(\xi) e^{t P(\xi)} \quad \text { for } \xi \in \mathbf{R}^{n}, t \in \mathbf{C}, \text { and } l \in \mathbf{N}_{0}
$$

where $Q_{j}(\xi)$ is an $N \times N$ matrix of polynomials of degree $\leq m(j+l)-|k|$, and $j_{0}$ is the least nonnegative integer such that $j_{0} \geq|k| / m-l$. For any $\varepsilon \in(0, \theta)$, let $t \in \Delta_{\theta-\varepsilon}$ and $l \in \mathbf{N}_{0}$. Then, by (2.2) and Lemma 2.1(b), there exists a constant $\omega_{\varepsilon}^{\prime}>0$ such that

$$
\left\|D^{k}\left(P(\xi)^{l} e^{t P(\xi)}\right)\right\| \leq \begin{cases}M_{\varepsilon}(\operatorname{Re} t)^{|k| / m-l} \exp \left(-\omega_{\varepsilon}^{\prime}|\xi|^{m} \operatorname{Re} t\right) & \text { for }|\xi| \geq L_{\varepsilon} \\ M_{\varepsilon}(\operatorname{Re} t)^{|k| / m-l} \exp \left(\omega_{\varepsilon}^{\prime} \operatorname{Re} t\right) & \text { for }|\xi|<L_{\varepsilon}\end{cases}
$$

where, and in the sequel, $M_{\varepsilon}$ denotes a generic constant depending on $\varepsilon$. Consequently

$$
\left\|D^{k}\left(P^{l} e^{t P}\right)\right\|_{L^{2}} \leq M_{\varepsilon}(\operatorname{Re} t)^{|k| / m-l}\left(\exp \left(\omega_{\varepsilon}^{\prime} \operatorname{Re} t\right)+(\operatorname{Re} t)^{-n / 2 m}\right) .
$$

Thus, by Bernstein's theorem, $P^{l} e^{t P} \in M_{N}\left(\mathcal{F} L^{1}\right)$ and

$$
\left\|P^{l} e^{t P}\right\|_{\mathcal{F} L^{1}} \leq M_{\varepsilon}(\operatorname{Re} t)^{-l} \exp \left(2 \omega_{\varepsilon}^{\prime} \operatorname{Re} t\right) \quad \text { for } t \in \Delta_{\theta-\varepsilon} \text { and } l \in \mathbf{N}_{0}
$$

Define $T(t)=\left(e^{t P}\right)(A)$ for $t \in \Delta_{\theta}$ and $T(0)=I_{N}$. Then (2.3) (with $l=0)$ implies that $\|T(t)\| \leq M_{\varepsilon} \exp \left(2 \omega_{\varepsilon}^{\prime} \operatorname{Re} t\right)\left(t \in \Delta_{\theta-\varepsilon}\right)$ and $T(t+s)=$ 
$T(t) T(s)\left(t, s \in \Delta_{\theta}\right)$. Also, (2.3) (with $l=1$ ) implies that $t \mapsto e^{t P} \in$ $H\left(\Delta_{\theta}, M_{N}\left(\mathcal{F} L^{1}\right)\right)$, and so $T(\cdot) \in H\left(\Delta_{\theta}, B\left(X^{N}\right)\right)$. Since for $\phi \in \mathcal{S}$

$$
\|T(t) \phi(A)-\phi(A)\| \leq M_{\varepsilon}|t| \exp \left(2 \omega^{\prime} \varepsilon \operatorname{Re} t\right)\|P \phi\|_{\mathcal{F} L^{1}} \rightarrow 0 \quad\left(\Delta_{\theta-\varepsilon} \ni t \rightarrow 0\right),
$$

the strong continuity of $T(t)\left(t \in \Delta_{\theta-\varepsilon} \cup\{0\}\right)$ follows from $\bar{E}=X$ and the estimate of $T(t)\left(t \in \Delta_{\theta-\varepsilon}\right)$. Thus $(T(t))_{t \in \Delta_{\theta}}$ is an analytic semigroup. To show that $\overline{P(A)}$ is its generator, let $L_{\lambda}$ for large $\lambda$ be the Laplace transform of $(T(t))_{t \geq 0}$. Then from Lemma 1.1(a) one has $P(A) T(t) \phi(A)=$ $T(t) P(A) \phi(A)$. Also, by Fubini's theorem,

$$
L_{\lambda}(\lambda-P(A)) \phi(A)=\left(\int_{0}^{\infty} e^{-\lambda t} e^{t P} d t(\lambda-P) \phi\right)(A)=\phi(A) .
$$

Thus Lemma 1.1(a) leads to $L_{\lambda}=R(\lambda, \overline{P(A)})$ for large $\lambda$. The claim now follows from [5, p. 627].

(a) Let $\omega_{1}<\omega_{2}<\omega$. Then by Lemma 2.1(b) there exist constants $\delta, L>0$ such that $\Lambda(P(\xi)) \leq-2 \delta|\xi|^{m}+\omega_{1}$ for $|\xi| \geq L$. It thus follows from (2.1) and (2.2) (with $l=0$ ) that

$$
\left\|D^{k} e^{t P(\xi)}\right\| \leq \begin{cases}M t^{|k| / m} \exp \left(-\delta|\xi|^{m} t+\omega_{2} t\right) & \text { for }|\xi| \geq L \text { and } t \geq 0 \\ M\left(t^{j_{0}}+t^{|k|}\right)\left(1+t^{N-1}\right) e^{\omega t} & \text { for }|\xi|<L \text { and } t \geq 0\end{cases}
$$

From this we obtain that

$$
\left\|D^{k} e^{t P}\right\|_{L^{2}} \leq M\left(t^{j_{0}}+t^{|k|}\right)\left(1+t^{N-1}\right) e^{\omega t}+M t^{(2|k|-n) / 2 m} e^{\omega_{0} t} \text { for } t>0 .
$$

Thus, by Bernstein's theorem, $e^{t P} \in M_{N}\left(\mathcal{F} L^{1}\right)$ and $\left\|e^{t P}\right\|_{\mathcal{F} L^{1}} \leq M(1+$ $\left.t^{N-1+n / 2}\right) e^{\omega t}$ for $t>0$, and so (a) follows.

(b) Let $w(\xi)=\left(1+|\xi|^{2}\right)^{\delta / 2}\left(\omega^{\prime}-P(\xi)\right)^{-1}$ for some $\omega^{\prime}>\omega$. Since (2.4) (with $k=0$ ) and the Hille-Yosida theorem lead to $\left\|\omega^{\prime}-P(\xi)\right\| \geq M|\xi|^{m}$ for $|\xi| \geq L$, it follows that $\left\|D^{k} w(\xi)\right\| \leq M|\xi|^{-|k|+\delta-m}$ for $|\xi| \geq L$ and $\leq M$ for $|\xi|<L$. Hence $w(A) \in B\left(X^{N}\right)$. Noting that (a) implies $\omega^{\prime} \in \rho(\overline{P(A)})$, one has $D(\overline{P(A)}) \subseteq\left(R\left(C_{\delta}\right)\right)^{N}$.

(c) We first show by induction that $D\left(\overline{P(A)}^{j(\mu)}\right) \subseteq\left(D\left(A^{\mu}\right)\right)^{N}$ for $\mu=$ $\left(\mu_{1}, \cdots, \mu_{n}\right) \in \mathbf{N}_{0}^{n}$, where $j(\mu)=\left[\frac{|\mu|}{m}\right]+1$. Let $v_{\mu}(\xi)=\xi^{\mu}\left(\omega^{\prime}-P(\xi)\right)^{-j(\mu)}$ for $\xi \in \mathbf{R}^{n}$. As seen above, we have $v_{\mu}(A) \in B\left(X^{N}\right)$. If $|\mu|=0$, then the statement is obvious. Let $\tilde{\mu}=\left(\mu_{1}, \cdots, \mu_{j}+1, \cdots, \mu_{n}\right)$ and, for any $x \in X^{N}$, choose $E^{N} \ni x_{k} \rightarrow x$. Then, by Lemma 1.1(a), $A_{j} A^{\mu} R\left(\omega^{\prime}, \overline{P(A)}\right)^{j(\tilde{\mu})} x_{k} \rightarrow$ $v_{\tilde{\mu}}(A) x$, Since $A_{j}$ is closed, the induction assumption yields $A^{\mu} R\left(\omega^{\prime}, \overline{P(A)}\right)^{j(\tilde{\mu})} x \in\left(D\left(A_{j}\right)\right)^{N}$, and thus the claim follows. Now we deduce from the properties of analytic semigroups that

$$
A^{\mu} T(t)=v_{\mu}(A) \sum_{k=0}^{j(\mu)}\left(\begin{array}{c}
j(\mu) \\
k
\end{array}\right)\left(\omega^{\prime}\right)^{j(\mu)-k}(-1)^{k} T^{(k)}(t) \quad \text { for } \mu \in \mathbf{N}_{0}^{n} \text { and } t \in \Delta_{\theta}
$$


and therefore (c) is proved.

Let $\mathrm{X}$ be some function space on which translations are uniformly bounded and strongly continuous. Then the above result can be applied to $P(D)$ (i.e., take $A=D$ ) on $X$, immediately. In the sequel, we assume that all partial differential operators (PDOs) have the maximal domains in the distributional sense, and so they are closed and densely defined on $X$. $X$ can be chosen as, for example, $L^{p}(1 \leq p<\infty),\left\{f \in C\left(\mathbf{R}^{n}\right) ; f\right.$ is bounded and uniformly continuous $\},\left\{f \in C\left(\mathbf{R}^{n}\right) ; \lim _{|x| \rightarrow \infty} f(x)=0\right\},\left\{f \in C\left(\mathbf{R}^{n}\right) ; f(x)\right.$ exists as $|x| \rightarrow \infty\},\left\{f \in C\left(\mathbf{R}^{n}\right) ; f\right.$ is 1-periodic $\}$, or $\left\{f \in C\left(\mathbf{R}^{n}\right) ; f\right.$ is almost periodic $\}$ with sup-norms. Moreover, let $W^{\alpha, X}(\alpha \geq 0)$ be the completion of $\mathcal{S}$ under the norm

$$
\|u\|_{\alpha, X} \equiv\|u\|_{X}+\left\|\mathcal{F}^{-1}\left(\left(1+|\cdot|^{2}\right)^{\alpha / 2} \mathcal{F} u\right)\right\|_{X} \quad \text { for } \quad u \in \mathcal{S} .
$$

When $X=L^{p}(1<p<\infty)$ and $\alpha \in \mathbf{N}_{0}, W^{\alpha, p} \equiv W^{\alpha, X}$ is the usual Sobolev space.

Corollary 2.3. Let $P(\xi)$ be parabolic in the sense of Petrovskij and $\theta$ be defined in Lemma 2.1. Then $P(D)$ generates an analytic semigroup $(T(t))_{t \in \Delta_{\theta}}$ on $X^{N}$, which satisfies Theorem $2.2(\mathrm{a})$ and $\left(W^{m+\delta, X}\right)^{N} \subseteq D(P(D)) \subseteq$ $\left(W^{m-\delta, X}\right)^{N}$ for any $\delta \in(0, m)$.

We remark that by Mihlin's multiplier theorem (see, e.g., [20, p. 96]) $D(P(D))=\left(W^{m, p}\right)^{N}$ if $X=L^{p}(1<p<\infty)$. We also remark that Theorem 2.2 can be applied to some PDOs with space-dependent coefficients and defined on bounded domains, such as on $L^{p}\left([0,1]^{n}\right)(1 \leq p<\infty)$, $\left\{f \in C\left([0,1]^{n}\right) ;\left.f\right|_{x_{j}=0}=\left.f\right|_{x_{j}=1}=0\right\}$, or $\left\{f \in C\left([0,1]^{n}\right) ;\left.f\right|_{x_{j}=0}=\left.f\right|_{x_{j}=1}\right\}$. For details we refer to $[\mathbf{1 5}, \mathbf{2 4}]$.

Example 2.4. Consider the following equation

$$
u_{t t}-2 a \Delta u_{t}-b \Delta u+c \Delta^{2} u=0
$$

where $a, b, c$ are positive constants. Then we can reduce it into the first order system associated with the matrix of polynomials

$$
P(\xi)=\left(\begin{array}{cc}
0 & -|\xi|^{2} \\
b+c|\xi|^{2} & -2 a|\xi|^{2}
\end{array}\right) .
$$

Since its principal part, i.e., $P_{2}(\xi)$ has eigenvalues $\left(-a \pm \sqrt{a^{2}-c}\right)|\xi|^{2}$, it follows from Corollary 2.3 that $P(D)$ generates an analytic semigroup $(T(t))_{t \in \Delta_{\theta}}$ on $X^{2}$, where

$$
\theta= \begin{cases}\pi / 2 & \text { for } a^{2} \geq c \\ \arcsin (a / \sqrt{c}) & \text { for } a^{2}<c\end{cases}
$$


Moreover, noting that $-a|\xi|^{2} \pm \sqrt{a^{2}|\xi|^{4}-b|\xi|^{2}-c|\xi|^{4}}$ are the eigenvalues of $P(\xi)$ one has that $\|T(t)\| \leq M\left(1+t^{1+n_{X}}\right)$ for $t \geq 0$.

\section{Parabolic $P(\xi)$ in the sense of Shilov.}

This section is concerned with the parabolic $\overline{P(A)}$ generating a regularized semigroup. Let $r \in(0, m], P(\xi)$ is said to be $r$-parabolic in the sense of Shilov [9] if there exist constants $\omega>0$ and $\omega^{\prime} \in \mathbf{R}$ such that $\Lambda(P(\xi)) \leq-\omega|\xi|^{r}+\omega^{\prime}$ for $\xi \in \mathbf{R}^{n}$. In particular, $P(\xi)$ is said to be Petrovskij correct [9] if $r=0$, i.e., $\sup _{\xi \in \mathbf{R}^{n}} \Lambda(P(\xi))<\infty$. By Lemma 2.1, $P(\xi)$ is parabolic in the sense of Petrovskij if and only if it is $m$-parabolic in the sense of Shilov.

The definitions of (exponentially bounded) regularized semigroups can be given by using Laplace transforms $[4,23]$. Let $C \in B(X)$ be injective. An exponentially bounded and strongly continuous family $(T(t))_{t \geq 0} \subseteq B(X)$ is called a $C$-regularized semigroup generated by a linear operator $B$ if $C^{-1} B C=B, \lambda-B$ is injective (for large $\lambda \in \mathbf{R}$ ), $R(C) \subseteq R(\lambda-B$ ), and $(\lambda-B)^{-1} C$ is the Laplace transform of $(T(t))_{t \geq 0}$. If, in addition, $T(\cdot) \in C([0, \infty), B(X)) \cap C^{\infty}((0, \infty), B(X))$ then $(T(t))_{t \geq 0}$ is said to be differentiable.

Theorem 3.1. Let $P(\xi)$ be r-parabolic in the sense of Shilov for some $r \in(0, m)$, and $\alpha>(m-r)(N-1+n / 2)$. Then $\overline{P(A)}$ generates a differentiable $C_{\alpha}$-regularized semigroup $(T(t))_{t \geq 0}$ on $X^{N}$, which satisfies the following statements.

(a) $\left\|T^{(l)}(t)\right\| \leq M M_{0}^{l}\left(\left(l ! t^{-l}\right)^{m / r}+t^{N-1+n / 2}\right) e^{\omega t}$ for $t \geq 0$ and $l \in \mathbf{N}_{0}$, where $M$ and $M_{0}$ are constants independent of $t$ and $l$, and $\omega=$ $\sup _{\xi \in \mathbf{R}^{n}} \Lambda(P(\xi))$.

(b) $D(\overline{P(A)}) \subseteq\left(R\left(C_{\delta}\right)\right)^{N}$, where $\delta=0$ if $r \leq \frac{n m}{n+2}$ and $0<\delta<r-n(m-$ $r) / 2$ if $r>\frac{n m}{n+2}$.

(c) $T(\cdot) \in C^{\infty}\left((0, \infty),\left(B\left(A^{\infty}\right)\right)^{N}\right)$.

Proof. By our assumption on $P(\xi)$, for $\omega^{\prime \prime}<\omega$ there exist constants $\omega^{\prime}$, $L>0$ such that

$$
\Lambda(P(\xi)) \leq-2 \omega^{\prime}|\xi|^{r}+\omega^{\prime \prime} \text { for }|\xi| \geq L
$$

Let $|k| \leq[n / 2]+1$. Then, by induction, there exists a constant $M_{1} \geq 0$ such that

$$
\left\|D^{k} P(\xi)^{l}\right\| \leq M_{1}^{l}|\xi|^{m l-|k|} \text { for }|\xi| \geq L \text { and } l \in \mathbf{N} \text {. }
$$


From (2.1) and (3.1) we have that

$$
\begin{aligned}
\left\|D^{k} e^{t P(\xi)}\right\| & \leq M \sum_{j=0}^{|k|} t^{j}|\xi|^{m j-|k|}\left(1+t+t|\xi|^{m}\right)^{N-1} \exp \left(-2 \omega^{\prime}|\xi|^{r} t+\omega^{\prime \prime} t\right) \\
& \leq M|\xi|^{(m-r-1)|k|+(m-r)(N-1)} \exp \left(-\omega^{\prime}|\xi|^{r} t+\omega t\right)
\end{aligned}
$$

for $|\xi| \geq L$ and $t \geq 0$, where, and in the sequel, $M$ denotes a generic constant independent of $l, t$ and $\xi$. Set $u_{t}=\left(1+|\cdot|^{2}\right)^{-\alpha / 2} e^{t P}$ for $t \geq 0$. It then follows from (3.2), (3.3), and Leibniz's formula that

$$
\begin{aligned}
\left\|D^{k}\left(P(\xi)^{l} u_{t}(\xi)\right)\right\| & \leq M M_{1}^{l}|\xi|^{(m-r-1)|k|+(m-r)(N-1)+l m-\alpha} \exp \left(-\omega^{\prime}|\xi|^{r} t+\omega t\right) \\
& \leq M M_{2}^{l}\left(l ! t^{-l}\right)^{m / r}|\xi|^{(m-r-1)|k|+(m-r)(N-1)-\alpha} e^{\omega t}
\end{aligned}
$$

for $|\xi| \geq L, t>0$ and $l \in \mathbf{N}_{0}$, where $M_{2}=M_{1}\left(r \omega^{\prime} / m\right)^{-m / r}$. Write $P^{l} u_{t}=$ $\left(u_{i j}^{l, t}\right)$, then we obtain by Lemma $1.1(\mathrm{c})$ that $u_{i j}^{l, t}\left(1-\psi_{i j}\right) \in \mathcal{F} L^{1}$ for some $\psi_{i j} \in C_{c}^{\infty}$, and

$$
\left\|u_{i j}^{l, t}\left(1-\psi_{i j}\right)\right\|_{\mathcal{F} L^{1}} \leq M M_{2}^{l}\left(l ! t^{-l}\right)^{m / r} e^{\omega t} \quad \text { for } t>0 \text { and } l \in \mathbf{N}_{0} .
$$

On the other hand, let $K>0$ such that $\operatorname{supp} \psi_{i j} \subset\left\{\xi \in \mathbf{R}^{n} ;|\xi| \leq K\right\}$ for $1 \leq i, j \leq N$. Since an induction implies that

$$
\left\|D^{k} P(\xi)^{l}\right\| \leq M_{3}^{l} \quad \text { for }|\xi| \leq K \text { and } l \in \mathbf{N},
$$

it follows from (2.2) and (2.1) that

$\left\|D^{k}\left(P(\xi)^{l} u_{t}(\xi)\right)\right\| \leq M M_{3}^{l}\left(1+t^{N-1+|k|}\right) e^{\omega t} \quad$ for $|\xi| \leq K, t \geq 0$ and $l \in \mathbf{N}_{0}$.

Thus Leibniz's formula and Bernstein's theorem lead to $u_{i j}^{l, t} \psi_{i j} \in \mathcal{F} L^{1}$ and

$$
\left\|u_{i j}^{l, t} \psi_{i j}\right\|_{\mathcal{F} L^{1}} \leq M M_{3}^{l}\left(1+t^{N-1+|k|}\right) e^{\omega t} \quad \text { for } t \geq 0 \text { and } l \in \mathbf{N}_{0} .
$$

Combining (3.4) and (3.6) one finds that $P^{l} u_{t} \in M_{N}\left(\mathcal{F} L^{1}\right)$ and

$$
\left\|P^{l} u_{t}\right\|_{\mathcal{F} L^{1}} \leq M M_{0}^{l}\left(\left(l ! t^{-l}\right)^{m / r}+t^{N-1+n / 2}\right) e^{\omega t} \quad \text { for } t>0 \text { and } l \in \mathbf{N}_{0},
$$

where $M_{0}=2 \max \left(M_{2}, M_{3}\right)$.

Define $T(t)=u_{t}(A)$ for $t \geq 0$. Here we note that when $l=0$, (3.4) is yet true for $t=0$. Furthermore, observing carefully the proof of (3.7) and using Lebesgue's dominated convergence theorem, one finds that $u_{t}(t \geq 0)$ is continuous in the norm $\|\cdot\|_{\mathcal{F} L^{1}}$, and so $T(\cdot) \in C([0, \infty), B(X))$. Obviously, (3.7) implies (a) and $T(\cdot) \in C^{\infty}((0, \infty), B(X))$, while $C_{\alpha}^{-1} \overline{P(A)} C_{\alpha}=\overline{P(A)}$ can be deduced from Lemma 1.1(a). The remainder of the proof may be 
carried out by modifying the corresponding parts of the proof of Theorem 2.2 .

We now turn to a stronger condition on $P(\xi)$. To this end, put

$$
\widetilde{\Lambda}(P)=\sup \left\{\operatorname{Re}(P y, y) ; y \in \mathbf{R}^{N} \text { and }\|y\|=1\right\} \quad \text { for } P \in M_{N}(\mathbf{C}),
$$

where $(\cdot, \cdot)$ is the inner product in $\mathbf{C}^{N}$ and $\|y\|=(y, y)^{1 / 2}$. Note that we also can write $\widetilde{\Lambda}(P)=\sup \{\operatorname{Re} z ; z \in$ n.r. $(P)\}$, where n.r. $(P)$ is the numerical range of $P$.

Theorem 3.2. Assume there exist constants $r \in(0, m), \omega^{\prime}>0$ and $\omega^{\prime \prime} \in \mathbf{R}$ such that

$$
\widetilde{\Lambda}(P(\xi)) \leq-\omega^{\prime}|\xi|^{r}+\omega^{\prime \prime} \quad \text { for } \xi \in \mathbf{R}^{n} .
$$

Let $\alpha>n(m-r) / 2$. Then $\overline{P(A)}$ generates a differentiable $C_{\alpha}$-regularized semigroup $(T(t))_{t \geq 0}$ on $X^{N}$ satisfying

$$
\left\|T^{(l)}(t)\right\| \leq M M_{0}^{l}\left(\left(l ! t^{-l}\right)^{m / r}+t^{n / 2}\right) e^{\omega t} \quad \text { for } t \geq 0 \text { and } l \in \mathbf{N}_{0},
$$

where $M$ and $M_{0}$ are constants independent of $t$ and $l$, and $\omega=$ $\sup _{\xi \in \mathbf{R}^{n}} \widetilde{\Lambda}(P(\xi))$.

Proof. By (3.8) there exist constants $\delta, L>0$ such that $\widetilde{\Lambda}(P(\xi)) \leq-\delta|\xi|^{r}+\omega$ for $|\xi| \geq L$. Thus the Lumer-Phillips theorem implies that

$$
\left\|e^{t P(\xi)}\right\| \leq \begin{cases}\exp \left\{-2 \omega_{1}|\xi|^{r} t+\omega t\right\} & \text { for }|\xi| \geq L \text { and } t \geq 0 \\ e^{\omega t} & \text { for }|\xi| \leq K \text { and } t \geq 0\end{cases}
$$

where $K>0$ is chosen as in the proof of Theorem 3.1. Set $u_{t}=(1+$ $\left.|\cdot|^{2}\right)^{-\alpha / 2} e^{t P}$ for $t \geq 0$. Then combining (2.2), (3.2), (3.5) and (3.9) yields that

$$
\left\|D^{k}\left(P(\xi)^{l} u_{t}(\xi)\right)\right\| \leq \begin{cases}M M_{2}^{l}\left(l ! t^{-l}\right)^{m / r}|\xi|^{(m-r-1)|k|-\alpha} e^{\omega t} & \text { for }|\xi| \geq L \\ M M_{3}^{l}\left(1+t^{|k|}\right) e^{\omega t} & \text { for }|\xi| \leq K,\end{cases}
$$

where $t>0$ and $l \in \mathbf{N}_{0}$. Now, the remainder of the proof may be copied from that of the proof of Theorem 3.1.

From the proof of Theorem 3.1 and 3.2 one finds that in the case $r=0$ the following result holds, which sharpens Theorem 3.1 and 4.2 in [2].

\section{Theorem 3.3.}

(a) Let $\omega \equiv \sup _{\xi \in \mathbf{R}^{n}} \Lambda(P(\xi))<\infty$ and $\alpha>m(N-1+n / 2)$. Then $\overline{P(A)}$ generates a $C_{\alpha}$-regularized semigroup $(T(t))_{t>0}$ on $X^{N}$ satisfying $T(\cdot) \in C\left([0, \infty),(B(X))^{N}\right)$ and $\|T(t)\| \leq M\left(1+t^{N-1+n / 2}\right) e^{\omega t}$ for $t \geq 0$. 
(b) Let $\omega \equiv \sup _{\xi \in \mathbf{R}^{n}} \widetilde{\Lambda}(P(\xi))<\infty$ and $\alpha>n m / 2$. Then $\overline{P(A)}$ generates a $C_{\alpha}$-regularized semigroup $(T(t))_{t \geq 0}$ on $X^{N}$ satisfying $T(\cdot) \in C([0, \infty)$, $\left.(B(X))^{N}\right)$ and $\|T(t)\| \leq M\left(1+t^{n / 2}\right) e^{\omega t}$ for $t \geq 0$.

Let $X$ be chosen as in the end of Section 2, and define

$$
n_{X} \begin{cases}=n\left|\frac{1}{2}-\frac{1}{p}\right| & \text { if } X=L^{p} \quad(1<p<\infty) \\ >n / 2 & \text { if } X=L^{1} \text { or the space of continuous functions. }\end{cases}
$$

Then the following holds.

\section{Corollary 3.4.}

(a) Let $P(\xi)$ be r-parabolic in the sense of Shilov for some $r \in(0, m)$ (resp. satisfy (3.8)). Then $P(D)$ generates a differentiable $R(1, \Delta)^{\alpha}-$ regularized semigroup on $X^{N}$, where $\alpha=(m-r)\left(N-1+n_{X}\right) / 2($ resp. $\left.=n_{X}(m-r) / 2\right)$.

(b) Let $\omega<\infty$, where $\omega$ is defined in Theorem 3.3(a) (resp. (b)). Then $P(D)$ generates a norm-continuous, $R(1, \Delta)^{\alpha}$-regularized semigroup on $X^{N}$, where $\alpha=m\left(N-1+n_{X}\right) / 2\left(\right.$ resp. $\left.=m n_{X} / 2\right)$. In particular, when $X=L^{2}$ we can choose $\alpha=m(N-1) / 2($ resp. $=0)$.

When $X$ is a space of continuous functions or $L^{1}$, Corollary 3.4 follows from Theorem 3.1-3.3, immediately. When $X=L^{p}(1<p<\infty)$, Corollary 3.4 can be deduced by modifying the proofs of Theorem 3.1-3.3. The main points are using the Riesz-Thorin convexity theorem and Miyachi's multiplier theorem $\mathrm{G}$ in [17] and noting $u(D) \phi=\mathcal{F}^{-1}(u \mathcal{F} \phi)$ for $u \in \mathcal{F} L^{1}$ and $\phi \in \mathcal{S}$. We refer to $[\mathbf{1 1}, \mathbf{2 4}]$ for the details. When $X=L^{2}$, the result (with $\alpha=$ $m(N-1) / 2$ ) was shown in [12], while the result (with $\alpha=0$ ) can be shown by modifying its proof (cf. the proof of Theorem 3.2). We remark that Corollary 3.4 (b) is essentially due to $[\mathbf{1 1}, \mathbf{1 2}]$. Moreover, when $X=\left\{f \in C\left(\mathbf{R}^{n}\right) ; f\right.$ is bounded $\}$ or $L^{\infty}$, one can show that Corollary 3.4 is still true for $n_{X}>n / 2$ (cf. [16]).

Example 3.5. (a) Consider the following linear system (cf. [1])

$$
\left\{\begin{array}{l}
u_{t}=2 a u_{x x}+b v_{x}-c v_{x x x} \\
v_{t}=u_{x} \\
u(0)=u_{0}, v(0)=v_{0}
\end{array}\right.
$$

where $a, b, c$ are positive constants. The corresponding matrix of polynomials is

$$
P(\xi)=\left(\begin{array}{cc}
-2 a \xi^{2} & i b \xi+i c \xi^{3} \\
i \xi & 0
\end{array}\right)
$$


By Theorem 4.14(e) in [7] $P(\xi)$ is not the generator of a strongly continuous semigroup on $\left(L^{1}(\mathbf{R})\right)^{2}$. Since the eigenvalues of $P(\xi)$ are $-a \xi^{2} \pm$ $\sqrt{a^{2} \xi^{4}-b \xi^{2}-c \xi^{4}}, P(\xi)$ is 2-parabolic in the sense of Shilov. It thus follows from Corollary 3.4(a) that $P(D)$ generates a differentiable $R(1, \Delta)^{\alpha}$ regularized semigroup on $\left(L^{1}(\mathbf{R})\right)^{2}$, where $\alpha>3 / 4$.

(b) We now reduce (2.5) with $c=0$ (cf. [2]) into the first order system (1.2) with

$$
P(\xi)=\left(\begin{array}{cc}
0 & 1 \\
-b|\xi|^{2} & -2 a|\xi|^{2}
\end{array}\right)
$$

Since the eigenvalues of $P(\xi)$ are $-a|\xi|^{2} \pm \sqrt{a^{2}|\xi|^{4}-b|\xi|^{2}}, P(\xi)$ is Petrovskij correct and satisfies that $\Lambda(P(\xi)) \leq 0$ for $\xi \in \mathbf{R}^{n}$. Thus Corollary 3.4(b) implies that $P(D)$ generates a norm-continuous, $R(1, \Delta)^{\alpha}$-regularized semigroup on $X^{2}$, where $\alpha=1+n_{X}$. Particularly, in the case $n=1$ we can choose $\alpha>3 / 2$, so that we obtain an improvement of the result in [2, Example 4.5], in which $\alpha>7 / 4$ is required.

Example 3.6. Consider the higher order Cauchy problem

$$
\begin{cases}u^{(N)}(t)=\sum_{k=1}^{N} p_{k}(A) u^{(k-1)}(t) & \text { for } t>0 \\ u^{(k-1)}(0)=u_{k} & \text { for } 1 \leq k \leq N\end{cases}
$$

on $X$, where $p_{k}(\xi)(1 \leq k \leq N)$ are polynomials of $\xi \in \mathbf{R}^{n}$. Let $\Lambda(\xi)=$ $\sup _{1 \leq j \leq N} \operatorname{Re} \lambda_{j}(\xi)$, where $\lambda_{j}(\xi)(1 \leq j \leq N)$ are the roots of the characteristic equation $\lambda^{N}=\sum_{k=1}^{N} p_{k}(\xi) \lambda^{k-1}$. Then (3.10) is said to be $r$-parabolic in the sense of Shilov [7, p. 218] if there exist constants $\omega>0$ and $\omega^{\prime} \in \mathbf{R}$ such that $\Lambda(\xi) \leq-\omega|\xi|^{r}+\omega^{\prime}$ for $\xi \in \mathbf{R}^{n}$. Write (3.10) as the system (1.1), in which

$$
\vec{u}(0)=\left(\begin{array}{c}
u_{1} \\
\vdots \\
u_{N}
\end{array}\right) \quad \text { and } \quad P(\xi)=\left(\begin{array}{ccccc}
0 & I & 0 & \cdots & 0 \\
\vdots & \ddots & \ddots & \ddots & \vdots \\
\vdots & & \ddots & \ddots & 0 \\
0 & \cdots & \cdots & 0 & I \\
p_{1}(\xi) & \cdots & \cdots & \cdots & p_{N}(\xi)
\end{array}\right) .
$$

Noting that

$$
\operatorname{det}(\lambda-P(\xi))=\lambda^{N}-\sum_{k=1}^{N} p_{k}(\xi) \lambda^{k-1},
$$

the $r$-parabolicity of (3.10) in the sense of Shilov is equivalent to that of $P(\xi)$. If (3.10) is $r$-parabolic in the sense of Shilov, then Theorem 3.1 and Lemma 1.2 imply that (3.10) has a unique solution $u \in C^{N-1}([0, \infty), X) \cap$ 
$C^{N}((0, \infty), X)$ for every $\vec{u}_{0} \in\left(R\left(C_{\alpha}\right)\right)^{N}$, where $\alpha>(m-r)(N-1+n / 2)$ and $m=\max \left\{\right.$ degree of $\left.p_{k}\right\}$. From this (2.5) has a unique solution $u \in$ $C^{1}([0, \infty), X) \cap C^{2}((0, \infty), X)$ for every initial value pair $\left(u(0, \cdot), u_{t}(0, \cdot)\right) \in$ $\left(W^{\alpha, X}\right)^{2}$, where $\alpha>2+n$. For general higher order Cauchy problems, we refer to, e.g., [18, 22].

\section{Systems with time-dependent coefficients.}

In this section we consider $P(t, \xi) \equiv \sum_{|\mu| \leq m} P_{\mu}(t) \xi^{\mu}$, where $P_{\mu} \in$ $C\left([0, T], M_{N}(\mathbf{C})\right)$ for $|\mu| \leq m$. Let $\Sigma, \widetilde{\Sigma}$ be some convex neighborhoods of $[0, T]$ in $\mathbf{C}$. Then we write $T_{\Delta}=\left\{(t, s) \in \mathbf{R}^{2} ; 0 \leq s<t \leq T\right\}$ and $\Sigma_{\theta}=\left\{(t, s) \in \Sigma^{2} ; t \neq s,|\arg (t-s)|<\theta\right\}$, where $\theta \in(0, \pi / 2]$, and denote by $\bar{T}_{\Delta}$ (resp. $\left.\bar{\Sigma}\right)$ the closure of $T_{\Delta}$ (resp. $\left.\Sigma\right)$. Moreover, $D_{t}=\partial / \partial t$.

Let $C \in B(X)$ be injective. A two parameter family $U(t, s) \in B(X)$, $(t, s) \in \bar{T}_{\Delta}$, is called a $C$-regularized evolution system if $U(t, r) U(r, s)=$ $U(t, s) C$ for $0 \leq s \leq r \leq t \leq T, U(t, t)=C$ for $0 \leq t \leq T$, and $U(\cdot, \cdot) x \in$ $C\left(\bar{T}_{\Delta}, X\right)$ for $x \in X$. In particular, an $I$-regularized evolution system is called an evolution system.

Theorem 4.1. Let $P(t, \xi)$ be parabolic in the sense of Petrovskij for every $t \in[0, T]$. Then there exists a unique evolution system $(U(t, s))_{(t, s) \in \bar{T}_{\Delta}}$ on $X^{N}$ such that

(a) $U(\cdot, \cdot) \in C^{1}\left(T_{\Delta},\left(B\left(A^{\infty}\right)\right)^{N}\right), \quad D_{t} U(t, s)=P(t, A) U(t, s)$ and $D_{s} U(t, s)=-P(s, A) U(t, s)$ for $(t, s) \in T_{\Delta}$.

(b) $\quad P_{\mu} \in C^{j}\left([0, T], M_{N}(\mathbf{C})\right)(|\mu| \leq m)$ for some $j \in \mathbf{N}$ implies $U(\cdot, \cdot) \in$ $C^{j+1}\left(T_{\Delta},\left(B\left(A^{\infty}\right)\right)^{N}\right)$. In particular $P_{\mu} \in C^{\infty}\left([0, T], M_{N}(\mathbf{C})\right)(|\mu| \leq m)$ implies $U(\cdot, \cdot) \in C^{\infty}\left(T_{\Delta},\left(B\left(A^{\infty}\right)\right)^{N}\right)$.

(c) $P_{\mu} \in H\left(\widetilde{\Sigma}, M_{N}(\mathbf{C})\right) \quad(|\mu| \leq m) \quad$ for some $\widetilde{\Sigma}$ implies $U(\cdot, \cdot) \in$ $H\left(\Sigma_{\theta},\left(B\left(A^{\infty}\right)\right)^{N}\right)$ for some $\Sigma_{\theta}$.

Proof. Since our assumptions on $P(t, \xi)$ imply that $\sup \{\Lambda(P(t, \xi)) ; \xi \in$ $\left.\mathbf{R}^{n}, t \in[0, T]\right\}<\infty$ and that there exist constants $\delta, L>0$ such that $\Lambda(P(t, \xi)) \leq-\delta|\xi|^{m}$ for $|\xi| \geq L$ and $t \in[0, T]$, the same argument as in the proof of Theorem 2.2 leads to $v_{t, s}^{\mu} \in \mathcal{F} L^{1}$ and

$$
\left\|v_{t, s}^{\mu}\right\|_{\mathcal{F} L^{1}} \leq M(t-s)^{-|\mu| / m} \quad \text { for } \quad(t, s) \in T_{\Delta},
$$

where $v_{t, s}^{\mu}(\xi)=\xi^{\mu} u_{t, s}(\xi)\left(\mu \in \mathbf{N}_{0}^{n}\right)$ and $u_{t, s}(\xi)=\exp \left\{\int_{s}^{t} P(\tau, \xi) d \tau\right\}$. Define $U(t, s)=u_{t, s}(A)$ for $(t, s) \in T_{\Delta}$ and $U(t, t)=I$ for $t \in[0, T]$. It easily follows from the properties of our functional calculus and (4.1) with $|\mu|=0$ that $(U(t, s))_{(t, s) \in \bar{T}_{\Delta}}$ is an evolution system on $X^{N}$, while the uniqueness follows from (a). 
(a) As shown in the proof of Theorem 2.2(b), one can deduce from (4.1) that $A^{\mu} U(t, s)=v_{t, s}^{\mu}(A) \in C\left(T_{\Delta}, B\left(X^{N}\right)\right)$. Similarly, from $D_{t} v_{t, s}^{\mu}=$ $P(t, \cdot) v_{t, s}^{\mu}$ and $D_{s} v_{t, s}^{\mu}=-P(s, \cdot) v_{t, s}^{\mu}$ for $(t, s) \in T_{\Delta}$ one has that $D_{t} A^{\mu} U(t, s)=$ $P(t, A) A^{\mu} U(t, s) \in C\left(T_{\Delta}, B\left(X^{N}\right)\right)$ and $D_{s} A^{\mu} U(t, s)=-P(s, A) A^{\mu} U(t, s) \in$ $C\left(T_{\Delta}, B\left(X^{N}\right)\right)$, respectively. Therefore we have the claim.

(b) We will show (b) by induction on $j \in \mathbf{N}_{0}$. When $j=0$, the statement has been showed in (a). Assume the statement is true for $k \leq j$. Then for any $j_{1}, j_{2} \in \mathbf{N}_{0}$ with $j_{1}+j_{2}=j+1$, it follows from the first equation in (a) and our assumptions that

$$
\begin{array}{rl}
D_{t}^{j_{1}+1} D_{s}^{j_{2}} & U(t, s) \\
& =\sum_{k_{1}+k_{2}=j_{1}}\left(\begin{array}{l}
j_{1} \\
k_{1}
\end{array}\right) D_{t}^{k_{1}} P(t, A) D_{t}^{k_{2}} D_{s}^{j_{2}} U(t, s) \in C\left(T_{\Delta},\left(B\left(A^{\infty}\right)\right)^{N}\right) .
\end{array}
$$

Similarly $D_{t}^{j_{1}} D_{s}^{j_{2}+1} U(t, s) \quad \in \quad C\left(T_{\Delta},\left(B\left(A^{\infty}\right)\right)^{N}\right)$, and so $U(\cdot, \cdot) \quad \in$ $C^{j+2}\left(T_{\Delta},\left(B\left(A^{\infty}\right)\right)^{N}\right)$.

(c) By the assumptions on $P(t, \xi)$ we have $\sup \left\{\Lambda(P(t, \xi)) ; \xi \in \mathbf{R}^{n}, t \in\right.$ $\Sigma\}<\infty$ for some $\Sigma$ with $\bar{\Sigma} \subseteq \widetilde{\Sigma}$. Then it follows from Theorem 2.2 that for every fixed $s \in \Sigma, \overline{P(s, A)}$ generates an analytic semigroup $(T(t, s))_{t \in \Delta_{\theta}}$ satisfying

$$
\|T(t, s)\| \leq M e^{\omega|t|} \quad \text { for } t \in \Delta_{\theta} \text { and } s \in \Sigma,
$$

where constants $\omega$ and $\theta \in(0, \pi / 2]$ can be chosen to be independent of $s$ (see the proof of Theorem 2.2).

On the other hand, set $v_{t}^{\mu}(\xi)=\xi^{\mu}\left(\omega^{\prime}-P(t, \xi)\right)^{-j(\mu)}$ for $t \in \Sigma$ and $\mu \in \mathbf{N}_{0}^{n}$, where $\omega^{\prime}>\omega$ and $j(\mu)=\left[\frac{|\mu|}{m}\right]+1$. Then a direct computation yields that $t \mapsto v_{t}^{\mu} \in H\left(\Sigma, M_{N}\left(\mathcal{F} L^{1}\right)\right)$. Thus, as proved in Theorem 2.2(c), we have

$$
A^{\mu} R\left(\omega^{\prime}, \overline{P(\cdot, A)}\right)^{j(\mu)} \in H\left(\Sigma, B\left(X^{N}\right)\right) \quad \text { for } \mu \in \mathbf{N}_{0}^{n} .
$$

In particular, $R\left(\omega^{\prime}, \overline{P(\cdot, A)}\right) \in H\left(\Sigma, B\left(X^{N}\right)\right)$. It now follows from this, (4.2), and [14, Theorem 1] (note also [21, Theorem 5.7.2]) that $U(\cdot, \cdot) \in$ $H\left(\Sigma_{\theta}, B\left(X^{N}\right)\right)$. Combining (4.3) with this we find $U(\cdot, \cdot) \quad \in$ $H\left(\Sigma_{\theta},\left(B\left(A^{\infty}\right)\right)^{N}\right)$.

The subsequent theorem can be showed by combining the proofs of Theorem 3.1, 3.2 and 4.1.

Theorem 4.2. Assume there exist constants $\delta>0, \omega \in \mathbf{R}$ and $r \in(0, m)$ such that $\Lambda(P(t, \xi))$ (resp. $\widetilde{\Lambda}(P(t, \xi))) \leq-\delta|\xi|^{r}+\omega$ for $\xi \in \mathbf{R}^{n}$ and $t \in[0, T]$. Let $\alpha>(m-r)(N-1+n / 2)($ resp. $>n(m-r) / 2)$. Then there exists a 
unique $C_{\alpha}$-regularized evolution system $(U(t, s))_{(t, s) \in \bar{T}_{\Delta}}$ on $X^{N}$ such that the conclusions $(a)$ and $(b)$ of Theorem 4.1 are still true.

Corresponding to Theorem 3.3 we have the following theorem, in which the numerical range part is related to an result in [4, Example 31.4].

Theorem 4.3. Let $\sup \{\Lambda(P(t, \xi))$ (resp. $\widetilde{\Lambda}(P(t, \xi))) ; \xi \in \mathbf{R}^{n}$ and $t \in$ $[0, T]\}<\infty$ and $\alpha>m(N-1+n / 2)$ (resp. $>n m / 2)$. Then there exists a unique $C_{\alpha}$-regularized evolution system $(U(t, s))_{(t, s) \in \bar{T}_{\Delta}}$ on $X^{N}$ such that $U(t, s):\left(R\left(C_{m}\right)\right)^{N} \rightarrow\left(R\left(C_{\beta}\right)\right)^{N}$ for some $\beta>m, U(\cdot, \cdot) x \in C^{1}\left(\bar{T}_{\Delta}, X^{N}\right)$, $D_{t} U(t, s) x=P(t, A) U(t, s) x$ and $D_{s} U(t, s) x=-P(s, A) U(t, s) x$ for $x \in$ $\left(R\left(C_{m}\right)\right)^{N}$ and $(t, s) \in \bar{T}_{\Delta}$.

Proof. As seen in the proof of Theorem 3.1 (resp. 3.2), we have that

$$
u_{t, s}^{\alpha} \equiv\left(1+|\cdot|^{2}\right)^{-\alpha / 2} \exp \left\{\int_{s}^{t} P(\tau, \cdot) d \tau\right\} \in M_{N}\left(\mathcal{F} L^{1}\right) \quad \text { for } \quad(t, s) \in \bar{T}_{\Delta} .
$$

It then is easy to check that $(U(t, s))_{(t, s) \in \bar{T}_{\Delta}}$ is a $C$-regularized evolution system on $X^{N}$, where $U(t, s)=u_{t, s}(A)$ for $(t, s) \in \bar{T}_{\Delta}$.

On the other hand, choose $m<\beta<m+\alpha-m(N-1+n / 2)$ (resp. $<$ $m+\alpha-m n / 2)$. Then $\delta \equiv m+\alpha-\beta>m(N-1+n / 2)$ (resp. $>m n / 2)$. It thus follows from $U(t, s) C_{m}=C_{\beta} u_{t, s}^{\delta}(A)$ that $U(t, s):\left(R\left(C_{m}\right)\right)^{N} \rightarrow\left(R\left(C_{\beta}\right)\right)^{N}$. Also, noting $R\left(C_{\gamma}\right) \subset D\left(A^{\mu}\right)$ for $|\mu|<\gamma$, one has that $U(t, s):\left(R\left(C_{m}\right)\right)^{N} \rightarrow$ $D(P(t, A))$ for $(t, s) \in \bar{T}_{\Delta}$. The desired equations now follow from this. Finally, the uniqueness of $(U(t, s))_{(t, s) \in \bar{T}_{\Delta}}$ can be proved by the standard method (cf. the proof of [15, Corollary 5.4]).

We now may apply Theorem 4.1-4.2 to the time-dependent system

$$
\begin{cases}\vec{u}_{t}(t, x)=P(t, D) \vec{u}(t, x) & \text { for } x \in \mathbf{R}^{n} \text { and } 0<t \leq T \\ \vec{u}(0, x)=\vec{u}_{0}(x) & \text { for } x \in \mathbf{R}^{n}\end{cases}
$$

on some function space $X$, for example, one of the spaces listed at the end of Section 2.

Corollary 4.4. Assume there exist constants $\delta>0, \omega \in \mathbf{R}$ and $r \in[0, m]$ such that $\Lambda(P(t, \xi))($ resp. $\widetilde{\Lambda}(P(t, \xi))) \leq-\delta|\xi|^{r}+\omega$ for $\xi \in \mathbf{R}^{n}$ and $t \in[0, T]$. Then (4.4) has a unique solution $\vec{u}(t, x)$ for every $\vec{u}_{0} \in\left(W^{\alpha, X}\right)^{N}$, where

$$
\alpha= \begin{cases}(m-r)\left(N-1+n_{X}\right)\left(\text { resp. } n_{X}(m-r)\right) & \text { if } r \in(0, m] \\ m\left(N+n_{X}\right)\left(\text { resp. } m\left(1+n_{X}\right)\right) & \text { if } r=0 .\end{cases}
$$


Here we note that Miklin's multiplier theorem implies $\left(W^{m, p}\right)^{N} \subseteq$ $D(P(t, D))$ for $t \in[0, T]$ and $1<p<\infty$.

Example 4.5. We consider the iterated evolution equation

$$
\begin{cases}\prod_{j=1}^{N}\left(D_{t}-\Delta-i p_{j}(t, D)\right) u(t, x)=0 & \text { for } x \in \mathbf{R}^{n} \text { and } 0<t \leq T \\ D_{t}^{j-1} u(0, x)=u_{j}(x) & \text { for } x \in \mathbf{R}^{n} \text { and } 1 \leq j \leq N\end{cases}
$$

on $L^{p}(1<p<\infty)$, where $p_{j}(t, \xi)=\sum_{|\mu| \leq m_{j}} p_{j \mu}(t) \xi^{\mu}$ and $p_{j \mu}(\cdot) \in$ $C([0, T], \mathbf{R})$ for $1 \leq j \leq N$. Then the roots of the characteristic equation of (4.5) are $\lambda_{j}(t, \xi)=-|\xi|^{2}+i p_{j}(t, \xi)$, and so $\operatorname{Re} \lambda_{j}(t, \xi)=-|\xi|^{2}$ for $t \in[0, T], \xi \in \mathbf{R}^{n}$ and $1 \leq j \leq N$. The same way as in Example 3.6 and Corollary 4.4 yield now that (4.5) has a unique solution $u \in$ $C^{N-1}\left([0, T], L^{p}\right) \cap C^{N}\left((0, T], L^{p}\right)$ for every $\left(u_{1}, \cdots, u_{N}\right) \in\left(W^{\alpha, p}\right)^{N}$, where $\alpha=\left(N-1+n\left|\frac{1}{2}-\frac{1}{p}\right|\right)\left(\sum_{j=1}^{N} \max \left(2, m_{j}\right)-2\right)$.

By Corollary 4.4 we easily generalize Example 3.6 to the case that the higher order Cauchy problem (3.10) has time-dependent cpefficients.

\section{References}

[1] J.L. Boldrini, Asymptotic behavior of traveling wave solutions of the equations for the flow of a fluid with small viscosity and capillarity, Quart. Appl. Math., 44 (1987), 697-708.

[2] R. deLaubenfels, Matrices of operators and regularized semigroups, Math. Z., 212 (1993), 619-629.

[3] _ Simultaneous well-posedness, Evolution Equation, Control Theory and Biomathematics: The Third International Conference, Hans-sur-Lesse 1991, Lect. Notes Pure Appl. Math., Vol. 155, Marcel Dekker (1993), 101-115.

[4] - Existence Families, Functional Calculi and Evolution Equations, Lect. Notes Math., Vol. 1570, Springer, Berlin, 1994.

[5] N. Dunford and J.T. Schwartz, Linear Operators, Part I: General Theory, Interscience, New York, 1960.

[6] Yu.V. Egorov and M.A. Shubin (eds), Partial Differential Equations II, SpringerVerlag, Berlin, 1994.

[7] K.-J. Engel, Operator Matrices and Systems of Evolution Equations, Book manuscript, 1995.

[8] A. Friedman, Generalized Functions and Partial Differential Equations, Prentice Hall, New York, 1963.

[9] I.M. Gelfand and G.E. Shilov, Generalized Functions, Vol 3, Academic Press, New York, 1967.

[10] M. Hieber, Integrated semigroups and the Cauchy problem for systems in $L^{p}$ spaces, J. Math. Anal. Appl., 162 (1991), 300-308.

[11] On linear hyperbolic systems with multiple characteristics, Differential Integral Equations, 8 (1995), 877-886. 
[12] M. Hieber, A. Holderrieth and F. Neubrander, Regularized semigroups and systems of linear partial differential equations, Ann. Scuola Norm. Sup. Pisa, 19 (1992), 363-379.

[13] L. Hörmander, Estimates for translation invariant operators in $L^{p}$ spaces, Acta Math., 104 (1960), 93-140.

[14] H. Komatsu, Abstract analyticity in time and unique continuation property of solutions of a parabolic equation, J. Fac. Sci. Univ. Tokyo, 9 (1961), 1-11.

[15] Y. Lei, W. Yi and Q. Zheng, Semigroups of operators and polynomials of generators of bounded strongly continuous groups, Proc. London Math. Soc., 69 (1994), 144170 .

[16] Y. Lei and Q. Zheng, The application of C-semigroups to differential operators in $L^{p}\left(R^{n}\right)$, J. Math. Anal. Appl., 188 (1994), 809-818.

[17] A. Miyachi, On some singular Fourier multipliers, J. Fac. Sci. Univ. Tokyo, 28 (1981), 267-315.

[18] F. Neubrander, Wellposedness of higher order abstract Cauchy problems, Trans. Amer. Math. Soc., 295 (1986), 257-290.

[19] S. Sjöstrand, On the Riesz means of the solutions of the Schrödinger equation, Ann. Scuola Norm. Sup. Pisa, 24 (1970), 331-348.

[20] E.M. Stein, Singular Integrals and Differentiability Properties of Functions, Princeton Univ. Press, New Jersey, 1970.

[21] H. Tanabe, Equations of evolution, Pitman, London, 1979.

[22] Q. Zheng, A Hille-Yosida theorem for the higher-order abstract Cauchy problem, Bull. London Math. Soc., 24 (1992), 531-539.

[23] Controllability of a class of linear systems in Banach space, Proc. Amer. Math. Soc., 123 (1995), 1241-1251.

[24] Cauchy problems for polynomials of generators of bounded $C_{0}$-groups and for differential operators, preprint, 1995.

Received May 20, 1996 and revised February 27, 1997. This project was supported by the National Science Foundation of China.

Huazhong Univ. of Science \& Technology

WUHAN 430074, P.R. OF CHINA

E-mail address: qzheng@server20.hust.edu.cn

Present address of the second author:

Institute of Applied Physics and Computational Mathematics

P.O. Box 8009-26, BeiJing 100088

P.R. OF CHINA 